\title{
Enhanced Technique via Filters for Seizure Prediction
}

\author{
Ahmed S*, El-Khobby H, Mahmoud A and Abd El-Samie FE \\ Electronics and Communications Department, Faculty of Engineering, Tanta University, Egypt
}

\begin{abstract}
This research study reports on the effective band of EEG signal to be used in seizure prediction, such as gamma, beta, alpha etc. The exercises were performed on a patient-specific framework for Electroencephalography (EEG) channel selection and seizure prediction, based on statistical probability distributions of the EEG signals. This framework is an enhanced method consists of two major phases, training and testing. Our objective was to distinguish between predicted and normal EEG signals. We achieved high prediction efficiency in reasonable time with low false alarm rate considering the parameters of seizure prediction techniques. Overall, we reached an efficiency of $96.2485 \%$ with prediction time of $54.012 \mathrm{~min}$ and false alarm rate of $0.10526 / \mathrm{h}$. This approach is having considerable significance. It is a simple method which depends on all filtering technique. This method can be implemented easily in future work and it doesn't have much computational load.
\end{abstract}

Keywords: EEG; Seizure prediction; Channel selection; Statistical analysis; EEG decomposition; Elliptic filter

\section{Introduction}

Berger et al. [1] inspired to continue exploration in EEG signal processing and associated direction. EEG signal processing has several applications either as medical applications such as seizure detection and prediction $[2,3]$ or non-medical applications including games and safety $[4,5]$. Epilepsy-related studies are the most interesting among these applications. Around $1 \%$ of the world population suffers from epilepsy and it is accompanied by seizures that can be traced by EEG [6]. The objective of this study was seizure prediction in EEG signals with suitable prediction time. Early anticipation of seizures can be used to alert to save patients from dangers. The efficiency of a seizure prediction algorithm is estimated with the prediction rate, false alarm rate, and prediction horizon [7-13].

Various researchers have investigated EEG seizure prediction using different techniques. Some of these efforts are tabulated in Table 1.

Empirical mode decomposition had been used in seizure prediction. For example, Qi et al. used EEG empirical mode decomposition (EMD) for seizure prediction with different prediction horizons [14]. They achieved a high sensitivity with time delays of 10-20 seconds. In addition, Principal Component Analysis (PCA) has been used as a pre-processing signal separation step for robust seizure prediction algorithms [15-17]. The main disadvantage of EMD is computation latency.

This paper presents a new time-domain approach for EEG channel selection and hence seizure prediction based on simple statistics with the efficient frequency band. The main idea was to discriminate between different signal activities based on their PDFs. Simulation experiments have proved that if non-overlapping segmentations of signals are traced, difference occurs to PDFs of segments even for the same category of segments. This means, PDF bins treatment could be made as random variables and selection of the most appropriate bins for discrimination can be done through a simple threshold process. The following section of the article gives a detailed explanation of the proposed approach followed by details of the pre-processing steps implemented prior to PDF calculation, EEG sub-banding and simulation results.

\section{Proposed Seizure Prediction Approach}

The proposed channel selection and seizure prediction approach depend mainly on estimating the PDFs of signal amplitude, derivative, local mean, local variance, and median of the different signal channels (Figure 1). Our approach consists of two phases; training and testing as illustrated (Figure 2). In the training phase, few hours were randomly selected for normal activities and two or three intervals for ictal and preictal activities. The selected periods with multi-channels are segmented into 10-second segments. Five PDFs were estimated, for each channel in each segment, (amplitude, derivative, local mean, local variance, and median).

Treatment for each PDF bin (9 bins) was done as a random variable for each of the normal, ictal, pre-ictal histogram classes. Based on predefined false alarm and prediction probability thresholds, the bins and the channels that discriminate between normal and pre-ictal classes were selected for discrimination in the testing phase.

\section{Pre-Processing of EEG Signals}

The proposed method depends on many pre-processing operations performed on the signal channels such as the derivative, local mean, local variance, and median. The derivative of an EEG signal reinforces the rapid transitions in the signals and degrades slow transitions. Hence, the different activities of the signal will yield distinguishable derivatives through their PDFs. The local mean is a good indication of the signal trend, and the local variance characterizes the signal power very well from sample to sample. The median filtering process removes any spikes that may have resulted from impulsive noise during the signal recording process. Based on the five estimated PDFs signal amplitude; derivative, local mean, local variance, and median for any EEG segment, discrimination can be done between normal, ictal, and pre-ictal signal segments.

*Corresponding author: Ahmed S, Electronics and Communications Department Faculty of Engineering, Tanta University, Egypt, Tel: +201226521508; E-mail: ahmed_sedik@f-eng.tanta.edu.eg

Received: December 07, 2016; Accepted: February 28, 2017; Published: March 05, 2017

Citation: Ahmed S, El-Khobby H, Mahmoud A, El-Samie AFE (2017) Enhanced Technique via Filters for Seizure Prediction. J Bioengineer \& Biomedical Sci 7: 218. doi: 10.4172/2155-9538.1000218

Copyright: ( 2017 Ahmed S, et al. This is an open-access article distributed under the terms of the Creative Commons Attribution License, which permits unrestricted use, distribution, and reproduction in any medium, provided the original author and source are credited. 
Citation: Ahmed S, El-Khobby H, Mahmoud A, El-Samie AFE (2017) Enhanced Technique via Filters for Seizure Prediction. J Bioengineer \& Biomedical Sci 7: 218. doi: 10.4172/2155-9538.1000218

Page 2 of 6

\begin{tabular}{|c|c|c|c|}
\hline Author & Method & Sensitivity/Efficiency & False alarm rate \\
\hline Parvez M and Paul M [7] & Customized phase correlation & $95 \%$ & 2.45 per patient \\
\hline Arabi [8] & Entropy & $90.20 \%$ & $0.11 / \mathrm{h}$ \\
\hline Shufang Li et al. [9] & Spike rate of interactional EEG & $75.80 \%$ & $0.09 / \mathrm{h}$ \\
\hline Wang et al. [10] & Reinforcement learning with online monitoring. & $70 \%$ & ------ \\
\hline Khalid Ml et al. [11] & LLE for EEG classification & $88 \%$ & ---- \\
\hline Hung et al. [12] & Wavelet transform & $87 \%$ & $0.24 / \mathrm{h}$ \\
\hline Gadhoumi et al. [13] & $\begin{array}{l}\text { Wavelet with measuring the similarity with a } \\
\text { reference EEG signal }\end{array}$ & $85 \%$ & $0.35 / h$ \\
\hline
\end{tabular}

Table 1: Some efforts of seizure prediction.

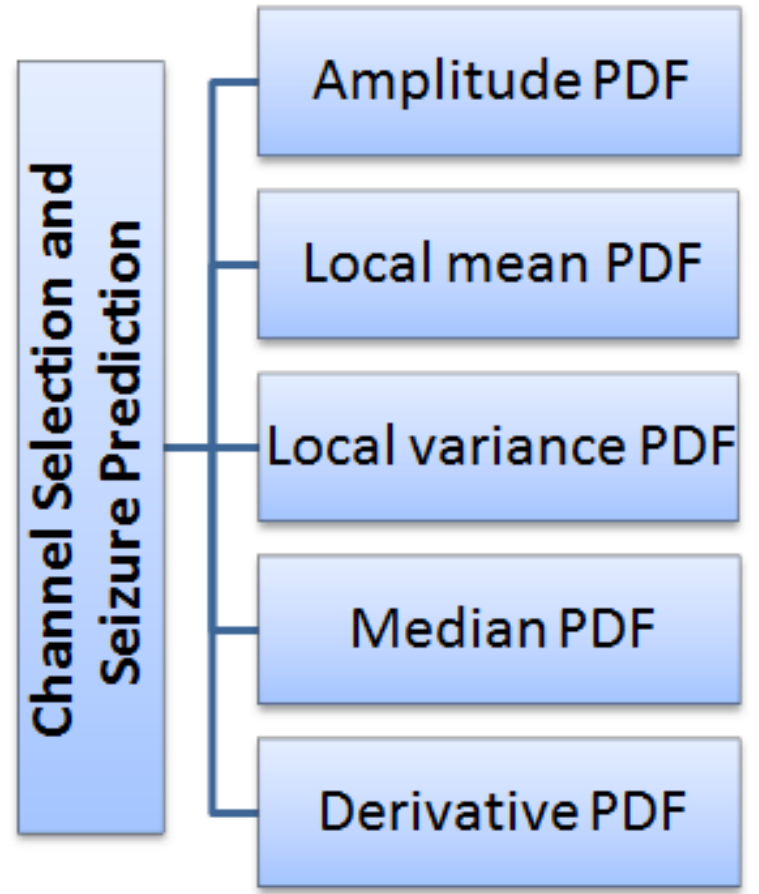

Figure 1: PDFs estimated from EEG signals for channel selection and seizure prediction.

\section{Signal derivative}

Abnormal activities in EEG signal are accompanied by sudden changes in amplitude. To reinforce these sudden changes, a signal differentiator can be utilized. We used a digital first-order differentiator filter for this purpose. This filter is given by [18]:

$$
H(\mathrm{z})=1-\mathrm{z}^{-1}
$$

\section{Local mean}

Local mean can be estimated for a signal $X(m)$ as follows [19]:

$$
X(m)=\frac{1}{(z i+1)} \sum_{i=m}^{m+i} X(i)
$$

While $(2 i+1)$ refers to the number of samples in the used segment for the estimation.

\section{Local variance}

Local variance can be estimated for a signal $X(m)$ as follows [20]:

$$
O_{x}^{\prime 2}(m)=\frac{1}{(2 i+1)} \sum_{i=m-i}^{m+i}(X(i)-X(m))^{2}
$$

\section{Median Filtering}

Median filtering is a sort of nonlinear smoothing operation of signals. It reduces some of the spikes in signals that may occur due to impulse noise. In the median filtering process, an odd set of signal samples were sorted. The middle value after sorting was extracted. For a median filter of length $N=2 i+1$, the filter output is given as [20]:

$$
Y(m)=\operatorname{MED}[X(m-i), \ldots, X(m), \ldots, X(m+i)]
$$

While, $X(m)$ and $Y(m)$ are the samples of the input and output sequences, respectively.

This type of median filtering is non-recursive so that an estimation of median filter output at any time is independent of the other results. Another type of median filtering is recursive. For a recursive median filter with window length $N=2 K+1$, the output is defined as [21]:

$$
Y(m)=\operatorname{MED}[Y(m-i), Y(m-i+1) \ldots, Y(m-1), X(m), \ldots, X(m+i)]
$$

This recursion process is a type of feedback that reduces noise more efficiently.

\section{Results}

Simulation experiments were carried out on 5 patients from MIT database (patients $1,8,11,14,20$ ) with 148.6133 hours containing 31 seizures for better understanding and assessment of the steps of the proposed approach [21].

Our main objective was to show the effective band of EEG signal that results in the best way to distinguish between normal and pre-ictal activities for early seizure prediction. We used the elliptic digital filter as a powerful filtering tool and display results. Elliptic digital band-pass filter, with order $\mathrm{K}=20$ and bandwidth $\mathrm{BW}$ from 0 to $30 \mathrm{~Hz}$ was used.

A moving average filter was used to refine the results (Figure 3), because a decision of a certain activity was not taken with a single signal segment. Multiple segments were required in the decision. Hence, the moving average process was appropriate for this action. The discrimination count on the vertical axis was compared with a selected threshold to determine the pre-ictal regions.

In the simulation experiments, we tested three different prediction horizons of 30,60 , and 90 minutes. In addition, a 15-minute postseizure horizon was adopted during the interpretation of the results. The obtained results for 5 MIT patients are tabulated in Table 2 .

\section{Discussion}

This paper shows the effect of adding an elliptic digital filter to the proposed channel selection approach as discussed previously. At this juncture, the objective was to calculate the seizure prediction parameters. These parameters were prediction time, false alarm rate and efficiency. 


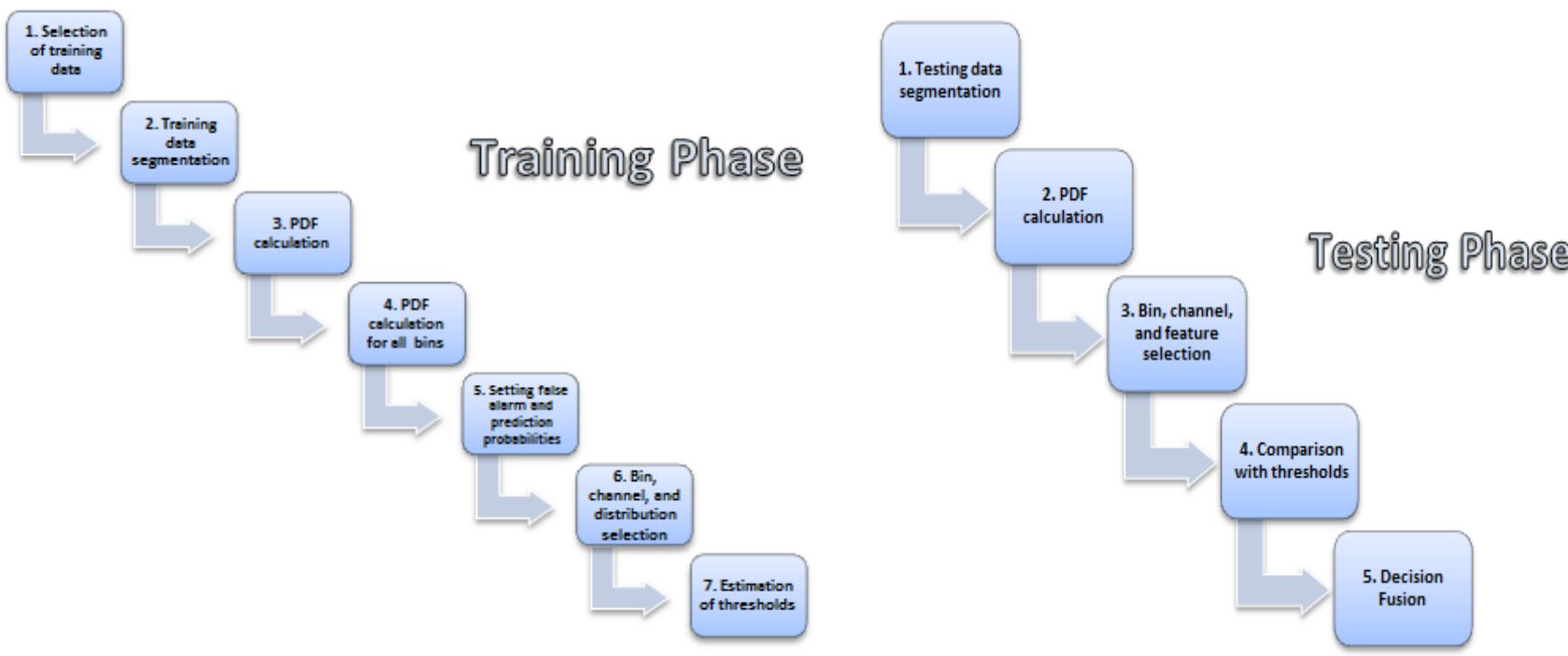

Figure 2: Training and testing phases of the proposed channel selection and seizure prediction algorithm.

(a)

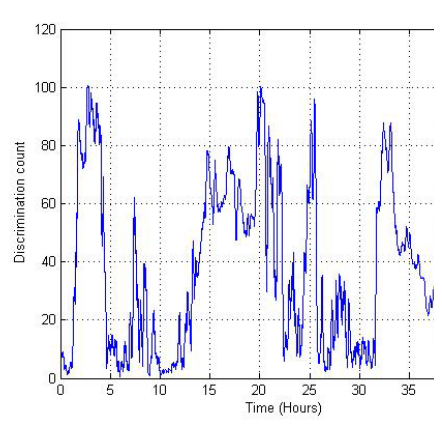

(c)

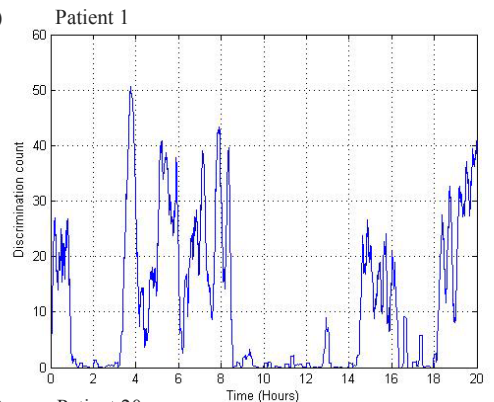

(e)

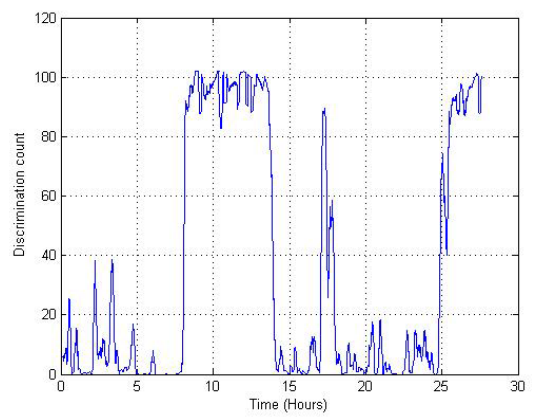

(b) Patient 8

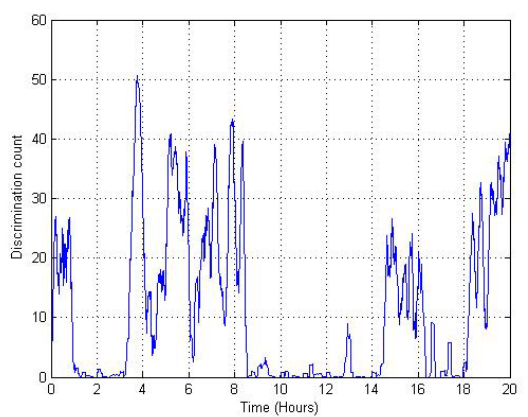

(d)

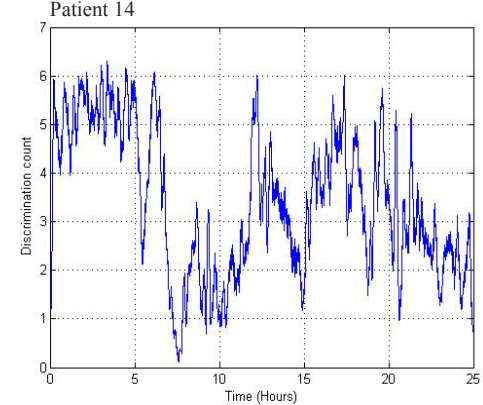

Figure 3: Variation of the discrimination count with time for the selected five patients with adding S-G digital smoothing filter. 
Citation: Ahmed S, El-Khobby H, Mahmoud A, El-Samie AFE (2017) Enhanced Technique via Filters for Seizure Prediction. J Bioengineer \& Biomedical Sci 7: 218. doi: 10.4172/2155-9538.1000218

Page 4 of 6

\begin{tabular}{|c|c|c|c|}
\hline Patient No. and data & 30 Minutes horizon & 60 Minutes horizon & 90 Minutes horizon \\
\hline $\begin{array}{c}\text { Patent No. } 1 \\
\text { Total No. of hours }=40.5522 \text { hours } \\
\text { Total No. of seizures }=7 \\
\text { Pred. prob. constraint }=70 \% \\
\text { False alarm prob. constraint }=30 \% \\
\text { Filter length }=60 \\
\text { Frequency band }=0-30 \mathrm{~Hz} \\
\text { Decision threshold }=75\end{array}$ & $\begin{array}{c}\text { No. of predicted seizures }=5 \\
\text { No. of false alarms }=8 \\
\text { Average prediction time }=(25.933333+30+30+27 \\
.2833333+29.3337) / 5=28.51 \mathrm{~min}\end{array}$ & $\begin{array}{c}\text { No. of predicted seizures }=6 \\
\text { No. of false alarms }=6 \\
\text { Average prediction time }=(25.933333 \\
+58.95+37.083333+43.5+27.28333+2 \\
9.39997) / 6=37.02499 \mathrm{~min})\end{array}$ & $\begin{array}{c}\text { No. of predicted seizures }=6 \\
\text { No. of false alarms }=5 \\
\text { Average prediction time }=(66.26667+90+3 \\
7.0833333+43.5+27.28333+29.36667) / 6= \\
48.91667 \mathrm{~min}\end{array}$ \\
\hline $\begin{array}{c}\text { Patent No. } 8 \\
\text { Total No. of hours }=20 \text { hours } \\
\text { Total No. of seizures }=5 \\
\text { Pred. prob. constraint }=70 \% \\
\text { False alarm prob. constraint }=30 \% \\
\text { Filter length }=60 \\
\text { Frequency band }=0-30 \mathrm{~Hz} \\
\text { Decision threshold }=26\end{array}$ & $\begin{array}{c}\text { No. of predicted seizures }=3 \\
\text { No. of false alarms }=3 \\
\text { Average prediction time } \\
(0+16.76667+30) / 3=15.58889 \mathrm{~min}\end{array}$ & $\begin{array}{c}\text { No. of predicted seizures }=5 \\
\text { No. of false alarms }=2 \\
\text { Average prediction time }(34.5+16.76 \\
667+46.3+55.11667+42.71667) / 5=3 \\
9.08 \text { min }\end{array}$ & $\begin{array}{c}\text { No. of predicted seizures }=5 \\
\text { No. of false alarms }=2 \\
\text { Average prediction time }=(34.5+16.76667+ \\
46.3+55.11667+42.71667) / 5=39.08 \mathrm{~min}\end{array}$ \\
\hline $\begin{array}{c}\text { Patent No. } 14 \\
\text { Total No. of hours }=25.851 \text { hours } \\
\text { Total No. of seizures }=8 \\
\text { Pred. prob. constraint }=60 \% \\
\text { False alarm prob. constraint }=40 \% \\
\text { Filter length }=60 \\
\text { Frequency band }=0-30 \mathrm{~Hz} \\
\text { Decision threshold }=5\end{array}$ & $\begin{array}{c}\text { No. of predicted seizures }=8 \\
\text { No. of false alarms }=7 \\
\text { Average prediction time }=(17.1+30+30+30+30+0 \\
.316667+23.65+18.55) / 8=22.45208 \mathrm{~min}\end{array}$ & $\begin{array}{c}\text { No. of predicted seizures }=8 \\
\text { No. of false alarms }=5 \\
\text { Average prediction time }=(58.433337 \\
+60+60+60+36.3+0.316667+23.65+3 \\
6.71667) / 8=41.92708 \mathrm{~min}\end{array}$ & $\begin{array}{c}\text { No. of predicted seizures }=8 \\
\text { No. of false alarms }=5 \\
\text { Average prediction time }=(76.433333+64.2 \\
+88.283333+90+90+0.316667+23.65+36.7 \\
1667) / 8=58.7 \mathrm{~min}\end{array}$ \\
\hline $\begin{array}{c}\text { Patent No. } 20 \\
\text { Total No. of hours }=27.595 \text { hours } \\
\text { Total No. of seizures }=8 \\
\text { Pred. prob. constraint }=70 \% \\
\text { False alarm prob. constraint }=30 \% \\
\text { Filter length }=60 \\
\text { Frequency band }=0-30 \mathrm{~Hz} \\
\text { Decision threshold }=100\end{array}$ & $\begin{array}{l}\text { No. of predicted seizures }=6 \\
\text { No. of false alarms }=0 \\
\text { Average prediction time }=(15.4+12.833333+30+ \\
30+10.48333+13.3333333) / 6=18.675 \mathrm{~min}\end{array}$ & $\begin{array}{l}\text { No. of predicted seizures }=8 \\
\text { No. of false alarms }=0 \\
\text { Average prediction time }=(15.4+12.8 \\
\begin{array}{c}33333+30.466667+56.01667+30+4 \\
4.4833333+48.433333+48.21667) / 8 \\
=35.73125 \mathrm{~min}\end{array}\end{array}$ & $\begin{array}{c}\text { No. of predicted seizures }=8 \\
\text { No. of false alarms }=0 \\
\text { Average prediction time }=(15.4+90+87.466 \\
67+81.683333+83.81667+44.4833333+79 . \\
43333+72.55) / 8=69.3517 \mathrm{~min}\end{array}$ \\
\hline
\end{tabular}


Citation: Ahmed S, El-Khobby H, Mahmoud A, El-Samie AFE (2017) Enhanced Technique via Filters for Seizure Prediction. J Bioengineer \& Biomedical Sci 7: 218. doi: 10.4172/2155-9538.1000218

Page 5 of 6

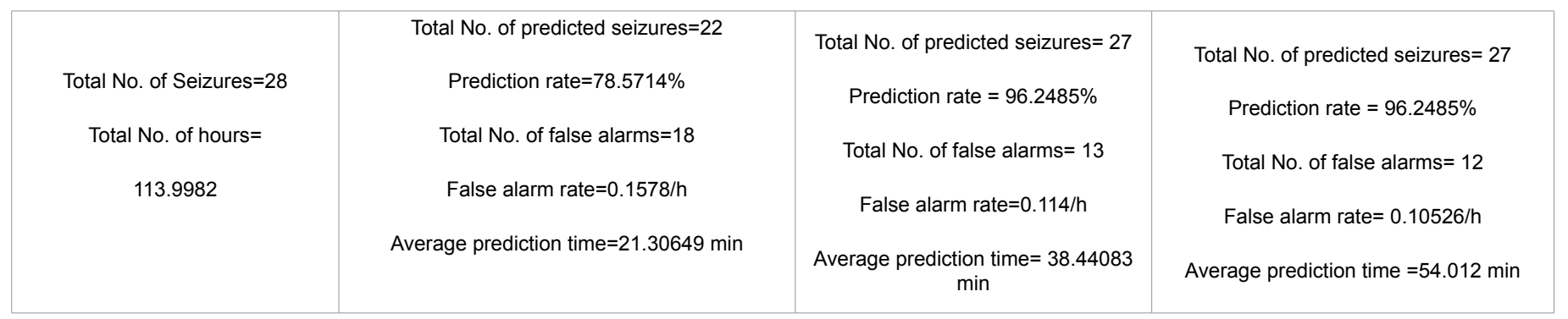

Table 2: Summary of the prediction results.

Prediction time is the time that system make an alarm to the patient that a predicted seizure would occur. It can be calculated as:

$$
T_{p}=T_{e}-T_{\text {offline }}
$$

Where $\mathrm{T}_{\mathrm{p}}$ is the prediction time, $\mathrm{T}_{\mathrm{e}}$ is the estimated prediction time due to the selected threshold and $\mathrm{T}_{\text {offline }}$ is the offline seizure time from the MIT database.

False alarm rate is the rate that system makes an alarm for a seizure and wouldn't occur. This false alarm can be calculated as:

$$
F A R=\frac{\text { No.of false alarms }}{\text { observation period }}
$$

The efficiency of proposed approach is the percentage of the predicted seizures overall observed seizures.

$$
\text { eff } .=\frac{\text { predicted seizures }}{\text { offline observed seizures }}
$$

These parameters are shown in the Table 2 with their respective values.

A brief comparison is shown in the Table 3 that shows we got an efficiency of $96.2485 \%$ which is comparatively better than previous efforts with suitable false alarm rate of $0.10526 / \mathrm{h}$. In addition, we achieved prediction time of $54.012 \mathrm{~min}$ which is suitable to generate the alarm for the patient and predict the seizure case in advance.

\section{Conclusion}

This paper presented an effective band of EEG signal with a statistical time-domain approach for EEG channel selection and seizure prediction depending on the estimation of the PDFs of the signals and the pre-processed versions. This approach is of multi-channel nature and it depends on pre-defined constraints for the required prediction and false alarm probabilities. Decision fusion and moving average post processing steps are utilized to enhance the prediction time and to achieve high prediction accuracies. The proposed method was utilized for different prediction horizons. It achieved prediction rates of $78.5714 \%, 96.2485 \%$, and $96.2485 \%$ respectively for prediction horizons of 30,60, and 90 minutes, with corresponding false alarm rates of $0.1578 / \mathrm{h}, 0.114 / \mathrm{h}$, and $0.10526 / \mathrm{h}$. The average prediction times were $21.30649 \mathrm{~min}, 38.44083 \mathrm{~min}$, and $54.012 \mathrm{~min}$ for the 30,60 , and 90 minutes horizons, respectively. These obtained results show that the proposed prediction method can be efficiently used for long prediction horizons.

\section{Compliance with Ethical Standards}

\section{Conflict of interest}

Ahmed Sedik, Heba El-Khobby, Mahmoud Atea, and Fathi Abd El-Samie declare that no conflict of interest.

\section{Ethical approval}

\begin{tabular}{|c|c|c|c|}
\hline Author & Method & $\begin{array}{l}\text { Sensitivity/ } \\
\text { Efficiency }\end{array}$ & False alarm rate \\
\hline $\begin{array}{c}\text { Parvez } M \text { and Paul } \\
\text { M [7] }\end{array}$ & $\begin{array}{c}\text { Customized phase } \\
\text { correlation }\end{array}$ & $95 \%$ & 2.45 per patient \\
\hline Arabi [8] & Entropy & $90.2 \%$ & $0.11 / \mathrm{h}$ \\
\hline $\begin{array}{l}\text { Shufang Li et } \\
\text { al. [9] }\end{array}$ & $\begin{array}{c}\text { Spike rate of } \\
\text { interactional EEG }\end{array}$ & $75.8 \%$ & $0.09 / \mathrm{h}$ \\
\hline Wang et al. [10] & $\begin{array}{l}\text { Reinforcement } \\
\text { learning with online } \\
\text { monitoring. }\end{array}$ & $70 \%$ & ----- \\
\hline Khalid MI et al. [11] & $\begin{array}{l}\text { LLE for EEG } \\
\text { classification }\end{array}$ & $88 \%$ & ---- \\
\hline Hung et al. [12] & Wavelet transform & $87 \%$ & $0.24 / \mathrm{h}$ \\
\hline $\begin{array}{c}\text { Gadhoumi et al. } \\
{[13]}\end{array}$ & $\begin{array}{l}\text { Wavelet with } \\
\text { measuring the } \\
\text { similarity with a } \\
\text { reference EEG } \\
\text { signal }\end{array}$ & $85 \%$ & $0.35 / \mathrm{h}$ \\
\hline Our work & $\begin{array}{l}\text { Statistical channel } \\
\text { selection with } \\
\text { adding digital filters }\end{array}$ & $96.2485 \%$ & $0.10526 / \mathrm{h}$ \\
\hline
\end{tabular}

This article does not contain any studies with human participants or animals performed by any of the authors.
Table 3: Comparison between our work and previous efforts.

\section{References}

1. Berger H (1929) Above the Elecrene Cephalogram of Man. Archive for Psychiatry and Nervous Diseases 87: 527-570.

2. Tzallas AT, Tsipouras MG, Tsalikakis DG, Karvounis EC, Astrakas L, et al. (2012) Automated Epileptic Seizure Detection Methods: A Review Study. Intech.

3. Alotaiby T, Alshebeili SA, Alshawi T, Ahmad I, Abd El-Samie FE (2014) EEG Seizure Detection and Prediction Algorithms: A survey. EURASIP Journal of Advances in Signal Processing.

4. Scherer R, Moitzi G, Daly I, Müller-Putz GR (2013) On the Use of Games for Noninvasive EEG-Based Functional Brain Mapping. IEEE Transactions on Computational Intelligence and Al in Games 5: 155-163.

5. Makeig S, Kothe C, Mullen T, Bigdely-Shamlo N, Zhang Z, et al. (2012) Evolving Signal Processing for Brain-Computer Interfaces. Proceedings of the IEEE 100: 1567-1584.

6. Thurman DJ, Beghi E, Begley CE, Berg AT, Buchhalter JR, et al. (2011) Standards for epidemiologic studies and surveillance of epilepsy. Epilepsia 52: 1-26.

7. Pezarv M, Paul M (2015) Epileptic Seizure Prediction by Exploiting Spatiotemporal Relationship of EEG Signals using Phase Correlation. IEEE Transactions on Neural Systems and Rehabilitation Engineering 24: 158-168.

8. Aarabi A, He B (123) A Rule-Based Seizure Prediction Method for Focal Neocortical Epilepsy. Clinical Neurophysiology 123: 1111-1122.

9. Zhou W, Yuan Q, Liu Y (2013) Seizure Prediction using Spike Rate of Intracranial EEG. IEEE transactions on Neural Systems and Rehabilitation Engineering 21: 880-886.

10. Wang S, Chaovalitwongse WA, Wong S (2010) A Novel Reinforcement Learning Framework for Online Adaptive Seizure Prediction. Proceedings of the IEEE International Conference on Bioinformatics and Biomedicine.

11. Khalid MI, Saeed A, Alshebeili SA, Abd El-Samie FE, Alotaiby T (2015) Online 
Citation: Ahmed S, El-Khobby H, Mahmoud A, El-Samie AFE (2017) Enhanced Technique via Filters for Seizure Prediction. J Bioengineer \& Biomedical Sci 7: 218. doi: 10.4172/2155-9538.1000218

Page 6 of 6

Adaptive Seizure Prediction Algorithm for Scalp EEG. IEEE International Conference on Information and Communication Technology Research.

12. Hung SH, Chao CF, Wang SK, Lin BS, Lin CT (2010) VLSI Implementation of Epileptic Seizure Prediction System based on Wavelet and Chaos Theory. TENCON 2010 - 2010 IEEE Region 10 Conference.

13. Gadhoumi K, Lina JM, Gotman J (2013) Seizure Prediction in Patients with Mesial Temporal Lobe Epilepsy using EEG Measures of State Similarity. Clinical Neurophysiology 124: 1745-1754.

14. Qi Y, Wang Y, Zheng X, Zhang J, Zhu J, et al. (2012) Efficient Epileptic Seizure Detection by a Combined IMF-VoE Feature. Proceedings of the International Conference of the IEEE EMBS.

15. Xie S, Krishnan S (2011) Signal Decomposition by Multi-scale PCA and Its Applications to Long-term EEG Signal Classification. Proceedings of the IEEE International Conference on Complex Medical Engineering.
16. Miri MR, Nasrabadi AM (2011) A New Seizure Prediction Method Based on Return Map. Proceedings of the Iranian Conference on BioMedical Engineering.

17. Williamson JR, Bliss DW, Browne DW, Narayanan JT (2012) Seizure Prediction using EEG Spatiotemporal Correlation Structure. Epilepsy \& Behavior 25: 230-238.

18. Kuo SM, Lee BH, Tian W (2006) Real-Time Digital Signal Processing: Implementations and Applications. John Wiley and Sons.

19. Abd El-Samie FE (2011) Information Security for Automatic Speaker Identification. Springer, Berlin. pp: 1-122.

20. Yin L, Yang R, Gabbouj M, Neuvo Y (1996) Weighted Median Filters: A Tutorial. IEEE Transactions on Circuits and Systems-II: Analog and Digital Signal Processing 43: 157-192.

21. http://physionet.org/pn6/chbmit/ 